\title{
INFLUENCE OF ADVERSE PRODUCTION FACTORS ON CEREBRAL HEMODYNAMIC IN LOCOMOTIVE DRIVERS
}

10.36740/WLek202012111

\author{
Olha M. Arustamian', Volodymyr S. Tkachyshyn', Vitalii E. Kondratiuk', Nataliia Yu. Tkachyshyna², \\ Oleksandr Yu. Aleksiichuk' ${ }^{1}$, Irina V. Dumka ${ }^{1}$ \\ 'BOGOMOLET'S NATIONAL MEDICAL UNIVERSITY, KYIV, UKRAINE \\ 2KYIV RAILWAY CLINICAL HOSPITAL №2 OF BRANCH «HEALTH CENTER» OF THE JOINT STOCK COMPANY «UKRAINIAN RAILWAY», KYIV, UKRAINE
}

\begin{abstract}
The aim: To investigate the pathological changes in intra- and extra cranial blood vessels in workers of locomotive crews depending on their length of service. Materials and methods: The study involved 249 male patients, aged from 21 to 60 years who worked as locomotive drivers. We used Doppler examination and studied Doppler Indexes of cerebral vessels.

Results: Results of examination showed that with increasing length of work of workers of locomotive crews (together with increasing of their age) we have established: 1) Reduction the elasticity of blood vessels (77.6\% of the patients);

2) Increased violation of venous circulation (86.4\%);

3) Reduced blood flow in the basilar artery (64\%);

4) Reduced blood flow in the right and left vertebral arteries ( $65.6 \%$ and $65.5 \%$ respectively);

5) Increase in the thickness of intimate / media complex more than 1.1 in $62 \%$ of drivers.

All of that also connected with length of their work.

Conclusions: we founded that speed of blood flow and elasticity in intra- and extra cranial vessels reduced with increase the length of service of workers of locomotive crews. Also we received such results: increasing violation of venous circulation, reducing blood flow in the basilar artery and reducing of blood flow in right and left vertebral arteries, increase intima / media thicknesses greater than 1,1 in locomotive drivers.
\end{abstract}

KEY WORDS: locomotive drivers, length of work, harmful production factors, Doppler examination, cerebral vessels

Wiad Lek. 2020;73(12 p. I):2617-2622

\section{INTRODUCTION}

The work of locomotive drivers is very difficult, intense and responsible. Action of harmful production factors on workers of locomotive crews has adverse impact on their health [1-5]. These factors leads to permanent stress and forced working position at the workplace, that characterized by excessive bending in the cervical spine. Nutrition of workers has irregular, unbalanced, irrational character, due to the shift work and the lack of normal conditions for nutrition in the cab of driver [6-8]. Workers of locomotive crews have most sedentary working posture and that is why they have pure physical activity. Also vibration, noise, electromagnetic radiation, unfavorable microclimate conditions, the effect of infrared radiation, neuro-emotional overload in driver's cabs act on health of workers $[9,10]$. Consequently, there is a high prevalence of somatic and occupational pathology among locomotive drivers, including vibration disease, neuro-sensory hearing loss, diseases of the cardiovascular system, digestive system, musculoskeletal system, visual organs and dust diseases of lungs [11-13]. A lots of production harmful factors leads to metabolic disorders and spreading of atherosclerotic changes and endothelium dysfunction in cerebral vessels [14]. Its manifested by increasing of the thickness of the carotid intima-media complex, the presence of atherosclerotic plaques, and decreased blood flow in the brain vessels Development of pathological depends on length of service of locomotive drivers [15-18].

Given that the pathogenesis of cardiovascular disease plays an important role in metabolic disorders, special attention should paid to the metabolic and disorders that occur among workers of locomotive crews $[19,20]$. Metabolic changes include hyperlipidemia, hyperglycemia and consequently the development of a number of pathological changes in the body, spreading of atherosclerotic changes in vessels of the circulatory system [21-24]. This article discusses of pathological changes that occur in intra and extra cranial vessels in locomotive drivers depending on their length of service [25]. The high information content and authenticity of extra- and intracranial Doppler examination provides an estimate hemodynamic significance of pathogenic factors of violation of circulation, degree of morphological changes, as well as a range of functional ability of arterial and venous circulation, when encephalopathy of various origins [26]. One of main reason of hemodynamic changes in cerebral 
Table 1. Indicators of Doppler examination of right part of cerebral vessels (averages and their error) in workers of locomotive crews $\mathrm{N}=249$

\begin{tabular}{|c|c|c|c|c|c|c|c|c|c|c|c|c|c|c|c|}
\hline \multicolumn{2}{|c|}{ a.car.com. } & \multicolumn{2}{|c|}{ a.car.ext. } & \multicolumn{2}{|c|}{ a.car.int. } & \multicolumn{2}{|c|}{ a.cer.ant. } & \multicolumn{2}{|c|}{ a.cer.post. } & \multicolumn{2}{|c|}{ a.cer.med. } & \multicolumn{2}{|c|}{ a.vert. } & \multicolumn{2}{|c|}{ a.basil. } \\
\hline $\begin{array}{c}\mathrm{LFV}(\mathrm{a}) \\
(\mathrm{cm} / \mathrm{sec})\end{array}$ & RI & $\begin{array}{c}\mathrm{LFV}(\mathrm{a}) \\
(\mathrm{cm} / \mathrm{sec})\end{array}$ & $\mathrm{RI}$ & $\begin{array}{c}\text { LFV(a) } \\
\text { (cm/sec) }\end{array}$ & RI & $\begin{array}{c}\mathrm{LFV}(\mathrm{m}) \\
(\mathrm{cm} / \mathrm{sec})\end{array}$ & RI & $\begin{array}{c}\mathrm{LFV}(\mathrm{m}) \\
(\mathrm{cm} / \mathrm{sec})\end{array}$ & $\mathrm{RI}$ & $\begin{array}{c}\mathrm{LFV}(\mathrm{m}) \\
(\mathrm{cm} / \mathrm{sec})\end{array}$ & RI & $\begin{array}{c}\text { LFV(a) } \\
(\mathrm{cm} / \mathrm{sec})\end{array}$ & RI & $\begin{array}{c}\mathrm{LFV}(\mathrm{a}) \\
(\mathrm{cm} / \mathrm{sec})\end{array}$ & RI \\
\hline $\begin{array}{c}26,45 \pm \\
0,39\end{array}$ & $\begin{array}{c}0,74 \pm \\
0,01\end{array}$ & $\begin{array}{c}29,3 \pm \\
0,43\end{array}$ & $\begin{array}{c}0,75 \pm \\
0,01\end{array}$ & $\begin{array}{c}27,04 \pm \\
0,63\end{array}$ & $\begin{array}{c}0,75 \pm \\
0,01\end{array}$ & $\begin{array}{c}72,25 \pm \\
0,41\end{array}$ & $\begin{array}{c}0,47 \pm \\
0,01\end{array}$ & $\begin{array}{c}64,46 \pm \\
0,59\end{array}$ & $\begin{array}{c}0,46 \pm \\
0,01\end{array}$ & $\begin{array}{c}81,57 \pm \\
0,74\end{array}$ & $\begin{array}{c}0,47 \pm \\
0,01\end{array}$ & $\begin{array}{c}28,84 \pm \\
0,72\end{array}$ & $\begin{array}{c}0,46 \pm \\
0,01\end{array}$ & $\begin{array}{c}31,06 \pm \\
0,7\end{array}$ & $\begin{array}{c}0,47 \pm \\
0,01\end{array}$ \\
\hline
\end{tabular}

Table 2. Indicators of Doppler examination of right part of cerebral vessels (averages and their error) in workers of locomotive crews $N=249$

\begin{tabular}{|c|c|c|c|c|c|c|c|c|c|c|c|c|c|c|c|}
\hline \multicolumn{2}{|c|}{ a.car.com. } & \multicolumn{2}{|c|}{ a.car.ext. } & \multicolumn{2}{|c|}{ a.car.int. } & \multicolumn{2}{|c|}{ a.cer.ant. } & \multicolumn{2}{|c|}{ a.cer.post. } & \multicolumn{2}{|c|}{ a.cer.med. } & \multicolumn{2}{|c|}{ a.vert. } & \multicolumn{2}{|c|}{ a.basil. } \\
\hline 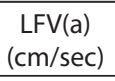 & $\mathrm{RI}$ & $\begin{array}{c}\mathrm{LFV}(\mathrm{a}) \\
(\mathrm{cm} / \mathrm{sec})\end{array}$ & $\mathrm{RI}$ & $\begin{array}{c}\mathrm{LFV}(\mathrm{a}) \\
(\mathrm{cm} / \mathrm{sec})\end{array}$ & RI & $\begin{array}{l}\mathrm{LFV}(\mathrm{m}) \\
(\mathrm{cm} / \mathrm{sec})\end{array}$ & $\mathrm{RI}$ & $\begin{array}{l}\mathrm{LFV}(\mathrm{m}) \\
(\mathrm{cm} / \mathrm{sec})\end{array}$ & $\mathrm{Rl}$ & $\begin{array}{l}\text { LFV(m). } \\
(\mathrm{cm} / \mathrm{sec})\end{array}$ & $\mathrm{RI}$ & $\begin{array}{c}\mathrm{LFV}(\mathrm{a}) \\
(\mathrm{cm} / \mathrm{sec})\end{array}$ & $\mathrm{RI}$ & $\begin{array}{c}\mathrm{LFV}(\mathrm{a}) \\
(\mathrm{cm} / \mathrm{sec})\end{array}$ & $\mathrm{RI}$ \\
\hline $\begin{array}{c}31,03 \pm \\
0,41\end{array}$ & $\begin{array}{c}0,76 \pm \\
0,01\end{array}$ & $\begin{array}{c}33,42 \pm \\
0,42\end{array}$ & $\begin{array}{c}0,75 \pm \\
0,01\end{array}$ & $\begin{array}{c}30,07 \pm \\
0,58\end{array}$ & $\begin{array}{c}0,76 \pm \\
0,01\end{array}$ & $\begin{array}{c}75,21 \pm \\
0,42\end{array}$ & $\begin{array}{c}0,47 \pm \\
0,01\end{array}$ & $\begin{array}{c}68,21 \pm \\
0,54\end{array}$ & $\begin{array}{c}0,46 \pm \\
0,1\end{array}$ & $\begin{array}{c}86,06 \pm \\
1,05\end{array}$ & $\begin{array}{c}0,47 \pm \\
0,01\end{array}$ & $\begin{array}{c}30,57 \pm \\
0,78\end{array}$ & $\begin{array}{c}0,46 \pm \\
0,01\end{array}$ & $\begin{array}{c}31,06 \pm \\
0,7\end{array}$ & $\begin{array}{c}0,47 \pm \\
0,01\end{array}$ \\
\hline
\end{tabular}

Table 3. Normal Doppler indicators of neck vessels and brain in control group aged from 40 to 60 years

\begin{tabular}{|c|c|c|c|c|c|c|c|c|c|c|c|c|c|c|c|}
\hline \multicolumn{2}{|c|}{ a.car.com. } & \multicolumn{2}{|c|}{ a.car.ext. } & \multicolumn{2}{|c|}{ a.car.int. } & \multicolumn{2}{|c|}{ a.cer.ant. } & \multicolumn{2}{|c|}{ a.cer.post. } & \multicolumn{2}{|c|}{ a.cer.med. } & \multicolumn{2}{|c|}{ a.vert. } & \multicolumn{2}{|c|}{ a.basil. } \\
\hline $\begin{array}{c}\text { LFV(a) } \\
(\mathrm{cm} / \mathrm{sec})\end{array}$ & RI & $\begin{array}{c}\text { LFV(a) } \\
(\mathrm{cm} / \mathrm{sec})\end{array}$ & RI & $\begin{array}{c}\text { LFV(a) } \\
(\mathrm{cm} / \mathrm{sec})\end{array}$ & RI & $\begin{array}{c}\text { LFV(m) } \\
(\mathrm{cm} / \mathrm{sec})\end{array}$ & RI & $\begin{array}{c}\text { LFV(m) } \\
(\mathrm{cm} / \mathrm{sec})\end{array}$ & RI & $\begin{array}{c}\mathrm{LFV}(\mathrm{m}) \\
(\mathrm{cm} / \mathrm{sec})\end{array}$ & RI & $\begin{array}{c}\text { LFV(a) } \\
(\mathrm{cm} / \mathrm{sec})\end{array}$ & $\mathrm{RI}$ & $\begin{array}{c}\text { LFV(a) } \\
(\mathrm{cm} / \mathrm{sec})\end{array}$ & RI \\
\hline $\begin{array}{l}19,9- \\
23,9\end{array}$ & $\begin{array}{c}0,63- \\
0,81\end{array}$ & $\begin{array}{c}24,7- \\
44,0\end{array}$ & $\begin{array}{c}0,73- \\
0,85\end{array}$ & $\begin{array}{c}24,0- \\
44,0\end{array}$ & $\begin{array}{c}0,55- \\
0,71\end{array}$ & $\begin{array}{c}85,4 \pm \\
20,1\end{array}$ & $\begin{array}{c}0,50 \pm \\
0,15\end{array}$ & $\begin{array}{c}60,1 \pm \\
20,6\end{array}$ & $\begin{array}{c}0,53 \pm \\
0,14\end{array}$ & $\begin{array}{l}91 \pm \\
16,9\end{array}$ & $\begin{array}{c}0,50 \pm \\
0,17\end{array}$ & $\begin{array}{c}36,4 \pm \\
11,7\end{array}$ & $\begin{array}{c}0,49 \pm \\
0,12\end{array}$ & $\begin{array}{c}36,4 \pm \\
11,7\end{array}$ & $\begin{array}{c}0,49 \pm \\
0,12\end{array}$ \\
\hline
\end{tabular}

Linear flow velocity (average) - LFV(a).

Linear flow velocity (maximum) - LFV(m).

Resistance index - Rl

vessels in workers of locomotive crews is prolonged flexion and extension in the cervical area during the work of machinists [27-29]. It leads to compression in the cervical spine and stenosis in the important arteries of the neck and head. In this article, the main goal is the study of brain and neck vessels using intra- and extra cranial Doppler examination, the identification and quantification of the degree of stenosis, atherosclerotic changes in workers of locomotive crews with complaints of transient ischemic attacks, and patients who have not clinical manifestations [30]. The main diagnostic unit during Doppler examination is Doppler spectrum. It can diagnosed pathological processes that lead to the development of local and systemic disorders of blood flow with corresponding Doppler equivalents [31, 32]. Given that a number of pathologic vascular processes accompanied by the development of similar hemodynamic violations received during examination Doppler indices, suggest the presence of patients with certain hemodynamic syndrome but not a specific disease [33]. Besides possible evaluation and dynamic monitoring of induced functional changes in blood flow and peripheral cerebral vasospasm, endothelium dysfunction and atherosclerotic changes of cranial and peripheral indicators reactivity [34]

The issue of early diagnosis and prevention of vascular diseases of the brain in working locomotive drivers are relevant for medicine rail transport because ischemic and atherosclerotic changes of vessels are established risk factors for brain strokes, vascular encephalopathy and sudden death [34].

\section{THE AIM}

The aim was to investigate the pathological changes in intra- and extra cranial blood vessels in workers of locomotive crews depending on their length of service.

\section{MATERALS AND METHODS}

The study involved examination of the main group that includes 249 males, aged 21 to 60 years who work as machinists and their assistants with an average age of 40.33 years. Among the workers in this category are $52.61 \%$ of smokers with 10 years or more. As a randomized by its basic parameters (sex and age) of the control group were employed 93 engineers (age from 40 to 60 years). This category of persons not exposed to harmful production factors. According to the work experience of the surveyed workers divided into 8 groups:

1) 1-5 years of work experience, 49 persons (19.7\%);

2) 6-10 years of work experience, 24 persons $(9.6 \%)$;

3) 11-15 years of work experience, 15 people $(6.0 \%)$;

4) $16-20$ years of work experience, 25 persons $(10.0 \%)$;

5) 21-25 years of work experience, 44 persons $(17.7 \%)$;

6) 26-30 years of work experience, 36 people (14.5\%);

7) 31-35 years of work experience, 29 persons (11.6\%);

8) 36-40 years of work experience, 27 persons (10.9\%).

Doppler ultrasound of the neck and brain (left and right departments) performed on a Vivid-7 machine manufactured by General Electric. From the Doppler ultrasound of the vessels of the brain and neck studied linear blood flow velocity (linear blood flow rate, linear maximum blood flow velocity), resistant index, the spread of reduced vascular elasticity, violation of venous circulation, reduced blood flow in the bloodstream. Excel applied software and specialized software STATGRAPHICS Plus v. 2.1. Study does not contradict the principles of the Declaration of Helsinki (2013), the European Convention on Human Rights and Biomedicine.

\section{RESULTS AND DISCUSSION}

In the course of the research calculated the average values and its errors of Doppler indicators of left and right 
Table 4. Indicators of Doppler of right intra- and extra cranial (averages and their error), depending on the length of service of workers of locomotive crews

\begin{tabular}{|c|c|c|c|c|c|c|c|c|c|c|c|c|c|c|c|c|c|}
\hline $\begin{array}{l}\text { Length } \\
\text { of work }\end{array}$ & $\begin{array}{l}N= \\
249\end{array}$ & a.car & om. & a.ca & xt. & a.car & int. & a.ce & nt. & a.cer. & ost. & a.cer. & ed. & a.v & & a.ba & \\
\hline & & $\begin{array}{c}\mathrm{LFV}(\mathrm{a}) \\
(\mathrm{cm} / \mathrm{sec})\end{array}$ & $\mathrm{RI}$ & $\begin{array}{c}\mathrm{LFV}(\mathrm{a}) \\
(\mathrm{cm} / \mathrm{sec})\end{array}$ & $\mathrm{RI}$ & $\begin{array}{c}\mathrm{LFV}(\mathrm{a}) \\
(\mathrm{cm} / \mathrm{sec})\end{array}$ & $\mathrm{RI}$ & $\begin{array}{c}\mathrm{LFV}(\mathrm{m}) \\
(\mathrm{cm} / \mathrm{sec})\end{array}$ & $\mathrm{RI}$ & $\begin{array}{c}\mathrm{LFV}(\mathrm{m}) \\
(\mathrm{cm} / \mathrm{sec})\end{array}$ & $\mathrm{RI}$ & $\begin{array}{c}\mathrm{LFV}(\mathrm{m}) \\
(\mathrm{cm} / \mathrm{sec})\end{array}$ & $\mathrm{RI}$ & $\begin{array}{c}\mathrm{LFV}(\mathrm{a}) \\
(\mathrm{cm} / \mathrm{sec})\end{array}$ & $\mathrm{RI}$ & $\begin{array}{c}\operatorname{LFV}(\mathrm{a}) \\
(\mathrm{cm} / \mathrm{sec})\end{array}$ & $\mathrm{Rl}$ \\
\hline $1-5$ & 49 & $\begin{array}{c}30,93 \\
\pm \\
1,04\end{array}$ & $\begin{array}{c}0,75 \\
\pm \\
0,01\end{array}$ & $\begin{array}{c}32,71 \\
\pm \\
1,22\end{array}$ & $\begin{array}{c}0,75 \\
\pm \\
0,01\end{array}$ & $\begin{array}{c}32,82 \\
\pm \\
1,49\end{array}$ & $\begin{array}{c}0,56 \\
\pm \\
0,01\end{array}$ & $\begin{array}{c}73,08 \\
\pm \\
0,86\end{array}$ & $\begin{array}{c}0,57 \\
\pm \\
0,01\end{array}$ & $\begin{array}{c}68,34 \\
\pm \\
1,3\end{array}$ & $\begin{array}{c}0,57 \\
\pm \\
0,01\end{array}$ & $\begin{array}{c}91,36 \\
\pm \\
1,96\end{array}$ & $\begin{array}{c}0,67 \\
\pm \\
0,01\end{array}$ & $\begin{array}{c}37,95 \\
\pm \\
23,7\end{array}$ & $\begin{array}{c}0,56 \\
\pm \\
0,01\end{array}$ & $\begin{array}{c}37,87 \\
\pm \\
2,66\end{array}$ & $\begin{array}{c}0,56 \\
\pm \\
0,01\end{array}$ \\
\hline 6-10 & 24 & $\begin{array}{c}30,47 \\
\pm \\
2,55\end{array}$ & $\begin{array}{c}0,75 \\
\pm \\
0,01\end{array}$ & $\begin{array}{c}32,27 \\
\pm \\
2,61\end{array}$ & $\begin{array}{c}0,76 \\
\pm \\
0,01\end{array}$ & $\begin{array}{c}28,55 \\
\pm \\
2,52\end{array}$ & $\begin{array}{c}0,75 \\
\pm \\
0,01\end{array}$ & $\begin{array}{c}71,75 \\
\pm \\
1,09\end{array}$ & $\begin{array}{c}0,56 \\
\pm \\
0,01\end{array}$ & $\begin{array}{c}65,48 \\
\pm \\
1,89\end{array}$ & $\begin{array}{c}0,56 \\
\pm \\
0,01\end{array}$ & $\begin{array}{c}87,15 \\
\pm \\
2,92\end{array}$ & $\begin{array}{c}0,67 \\
\pm \\
0,01\end{array}$ & $\begin{array}{c}32,02 \\
\pm \\
2,93\end{array}$ & $\begin{array}{c}0,56 \\
\pm \\
0,01\end{array}$ & $\begin{array}{c}34,8 \\
\pm \\
2,94\end{array}$ & $\begin{array}{c}0,58 \\
\pm \\
0,01\end{array}$ \\
\hline $11-15$ & 15 & $\begin{array}{c}29,55 \\
\pm \\
1,3\end{array}$ & $\begin{array}{c}0,74 \\
\pm \\
0,01\end{array}$ & $\begin{array}{c}31,02 \\
\pm \\
1,9\end{array}$ & $\begin{array}{c}0,76 \\
\pm \\
0,01\end{array}$ & $\begin{array}{c}28,33 \\
\pm \\
2,17\end{array}$ & $\begin{array}{c}0,77 \\
\pm \\
0,01\end{array}$ & $\begin{array}{c}70,26 \\
\pm \\
1,33\end{array}$ & $\begin{array}{c}0,58 \\
\pm \\
0,01\end{array}$ & $\begin{array}{c}65,42 \\
\pm \\
1,9\end{array}$ & $\begin{array}{c}0,58 \\
\pm \\
0,01\end{array}$ & $\begin{array}{c}84,77 \\
\pm \\
1,34\end{array}$ & $\begin{array}{c}0,68 \\
\pm \\
0,01\end{array}$ & $\begin{array}{c}30,08 \\
\pm \\
2,1\end{array}$ & $\begin{array}{c}0,56 \\
\pm \\
0,01\end{array}$ & $\begin{array}{c}31,4 \\
\pm \\
2,07\end{array}$ & $\begin{array}{c}0,56 \\
\pm \\
0,01\end{array}$ \\
\hline $16-20$ & 25 & $\begin{array}{c}27,2 \\
\pm \\
0,65\end{array}$ & $\begin{array}{c}0,74 \\
\pm \\
0,01\end{array}$ & $\begin{array}{c}29,82 \\
\pm \\
0,96\end{array}$ & $\begin{array}{c}0,76 \\
\pm \\
0,01\end{array}$ & $\begin{array}{c}27,41 \\
\pm \\
1,44\end{array}$ & $\begin{array}{c}0,76 \\
\pm \\
0,01\end{array}$ & $\begin{array}{c}68,71 \\
\pm \\
0,88\end{array}$ & $\begin{array}{c}0,58 \\
\pm \\
0,01\end{array}$ & $\begin{array}{c}63,7 \\
\pm \\
1,02\end{array}$ & $\begin{array}{c}0,57 \\
\pm \\
0,01\end{array}$ & $\begin{array}{c}82,28 \\
\pm \\
2,03\end{array}$ & $\begin{array}{c}0,58 \\
\pm \\
0,01\end{array}$ & $\begin{array}{c}28,27 \\
\pm \\
1,48\end{array}$ & $\begin{array}{c}0,57 \\
\pm \\
0,01\end{array}$ & $\begin{array}{c}29,01 \\
\pm \\
1,66\end{array}$ & $\begin{array}{c}0,56 \\
\pm \\
0,01\end{array}$ \\
\hline $21-25$ & 44 & $\begin{array}{c}25,38 \\
\pm \\
0,72\end{array}$ & $\begin{array}{c}0,64 \\
\pm \\
0,01\end{array}$ & $\begin{array}{c}28.34 \\
\pm \\
0,76\end{array}$ & $\begin{array}{c}0,76 \\
\pm \\
0,01\end{array}$ & $\begin{array}{c}23,54 \\
\pm \\
1,11\end{array}$ & $\begin{array}{c}0,56 \\
\pm \\
0,01\end{array}$ & $\begin{array}{c}67,89 \\
\pm \\
0,32\end{array}$ & $\begin{array}{c}0,46 \\
\pm \\
0.01\end{array}$ & $\begin{array}{c}62,77 \\
\pm \\
0,72\end{array}$ & $\begin{array}{c}0,46 \\
\pm \\
0,01\end{array}$ & $\begin{array}{c}77,19 \\
\pm \\
0,66\end{array}$ & $\begin{array}{c}0,45 \\
\pm \\
0,01\end{array}$ & $\begin{array}{c}24,91 \\
\pm \\
0,97\end{array}$ & $\begin{array}{c}0,43 \\
\pm \\
0,01\end{array}$ & $\begin{array}{c}27,78 \\
\pm \\
0,73\end{array}$ & $\begin{array}{c}0,43 \\
\pm \\
0,01\end{array}$ \\
\hline $26-30$ & 36 & $\begin{array}{c}24,83 \\
\pm \\
0,57\end{array}$ & $\begin{array}{c}0,65 \\
\pm \\
0,01\end{array}$ & $\begin{array}{c}28,15 \\
\pm \\
0,64\end{array}$ & $\begin{array}{c}0,73 \\
\pm \\
0,01\end{array}$ & $\begin{array}{c}23,02 \\
\pm \\
0,84\end{array}$ & $\begin{array}{c}0,56 \\
\pm \\
0,01\end{array}$ & $\begin{array}{c}67,63 \\
\pm \\
0,67\end{array}$ & $\begin{array}{c}0,46 \\
\pm \\
0,01\end{array}$ & $\begin{array}{c}62,21 \\
\pm \\
0,68\end{array}$ & $\begin{array}{c}0,43 \\
\pm \\
0,01\end{array}$ & $\begin{array}{c}76,81 \\
\pm \\
0,82\end{array}$ & $\begin{array}{c}0,45 \\
\pm \\
0,01\end{array}$ & $\begin{array}{c}24,33 \\
\pm \\
0,78\end{array}$ & $\begin{array}{c}0,44 \\
\pm \\
0,01\end{array}$ & $\begin{array}{c}26,86 \\
\pm \\
0,62\end{array}$ & $\begin{array}{c}0,43 \\
\pm \\
0,01\end{array}$ \\
\hline $31-35$ & 29 & $\begin{array}{c}24,86 \\
\pm \\
0,7\end{array}$ & $\begin{array}{c}0,64 \\
\pm \\
0,01\end{array}$ & $\begin{array}{c}27,02 \\
\pm \\
0,4\end{array}$ & $\begin{array}{c}0,73 \\
\pm \\
0,01\end{array}$ & $\begin{array}{c}21,84 \\
\pm \\
0,48\end{array}$ & $\begin{array}{c}0,55 \\
\pm \\
0,01\end{array}$ & $\begin{array}{c}67,09 \\
\pm \\
1,15\end{array}$ & $\begin{array}{c}0,45 \\
\pm \\
0,01\end{array}$ & $\begin{array}{c}61,86 \\
\pm \\
1,31\end{array}$ & $\begin{array}{c}0,43 \\
\pm \\
0,01\end{array}$ & $\begin{array}{c}76,13 \\
\pm \\
0,46\end{array}$ & $\begin{array}{c}0,43 \\
\pm \\
0,01\end{array}$ & $\begin{array}{c}24,02 \\
\pm \\
0,66\end{array}$ & $\begin{array}{c}0,43 \\
\pm \\
0,01\end{array}$ & $\begin{array}{c}26,45 \\
\pm \\
0,41\end{array}$ & $\begin{array}{c}0,43 \\
\pm \\
0,01\end{array}$ \\
\hline $36-40$ & 27 & $\begin{array}{c}24,36 \\
\pm \\
0,53\end{array}$ & $\begin{array}{c}0,84 \\
\pm \\
0,01\end{array}$ & $\begin{array}{c}27,01 \\
\pm \\
0,73\end{array}$ & $\begin{array}{c}0,85 \\
\pm \\
0,01\end{array}$ & $\begin{array}{c}21,2 \\
\pm \\
0,66\end{array}$ & $\begin{array}{c}0,76 \\
\pm \\
0,01\end{array}$ & $\begin{array}{c}67,03 \\
\pm \\
0,33\end{array}$ & $\begin{array}{c}0,56 \\
\pm \\
0,01\end{array}$ & $\begin{array}{c}60,96 \\
\pm \\
0,75\end{array}$ & $\begin{array}{c}0,56 \\
\pm \\
0,01\end{array}$ & $\begin{array}{c}73,66 \\
\pm \\
2,13\end{array}$ & $\begin{array}{c}0,57 \\
\pm \\
0,01\end{array}$ & $\begin{array}{c}24,01 \\
\pm \\
0,76\end{array}$ & $\begin{array}{c}0,56 \\
\pm \\
0,01\end{array}$ & $\begin{array}{c}26,1 \\
\pm \\
0,85\end{array}$ & $\begin{array}{c}0,58 \\
\pm \\
0,01\end{array}$ \\
\hline
\end{tabular}

Table 5. Indicators of Doppler of left intra- and extra cranial (averages and their error), depending on the length of service of workers of locomotive crews

\begin{tabular}{|c|c|c|c|c|c|c|c|c|c|c|c|c|c|c|c|c|c|}
\hline $\begin{array}{l}\text { Length of } \\
\text { work }\end{array}$ & $\begin{array}{l}N= \\
249\end{array}$ & a.car.c & & a.car.e & & a.car. & & a.cer.a & & a.cer.p & & a.cer.m & ed. & a.ver & & a.bas & \\
\hline & & $\begin{array}{c}\mathrm{LFV}(\mathrm{a}) \\
(\mathrm{cm} / \mathrm{sec})\end{array}$ & $\mathrm{RI}$ & $\begin{array}{c}\mathrm{LFV}(\mathrm{a}) \\
(\mathrm{cm} / \mathrm{sec})\end{array}$ & $\mathrm{RI}$ & $\begin{array}{c}\operatorname{LFV}(\mathrm{a}) \\
(\mathrm{cm} / \mathrm{sec})\end{array}$ & $\mathrm{RI}$ & $\begin{array}{c}\mathrm{LFV}(\mathrm{m}) \\
(\mathrm{cm} / \mathrm{sec})\end{array}$ & RI & 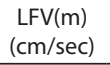 & $\mathrm{RI}$ & $\begin{array}{c}\operatorname{LFV}(\mathrm{m}) \\
(\mathrm{cm} / \mathrm{sec})\end{array}$ & RI & $\begin{array}{c}\operatorname{LFV(a)} \\
(\mathrm{cm} / \mathrm{sec})\end{array}$ & $\mathrm{RI}$ & $\begin{array}{c}\operatorname{LFV(a)} \\
(\mathrm{cm} / \mathrm{sec})\end{array}$ & $\mathrm{RI}$ \\
\hline $1-5$ & 49 & $\begin{array}{c}32,96 \\
\pm \\
1,19 \\
\end{array}$ & $\begin{array}{c}0,75 \\
\pm \\
0,01\end{array}$ & $\begin{array}{c}36,91 \\
\pm \\
1,41\end{array}$ & $\begin{array}{c}0,76 \\
\pm \\
0,01\end{array}$ & $\begin{array}{c}35,92 \\
\pm \\
1,75\end{array}$ & $\begin{array}{c}0,77 \\
\pm \\
0,01\end{array}$ & $\begin{array}{c}78,17 \\
\pm \\
0,99\end{array}$ & $\begin{array}{c}0,57 \\
\pm \\
0,01\end{array}$ & $\begin{array}{c}72,12 \\
\pm \\
1,46\end{array}$ & $\begin{array}{c}0,58 \\
\pm \\
0,01\end{array}$ & $\begin{array}{c}96,5 \\
\pm \\
1,82 \\
\end{array}$ & $\begin{array}{c}0,67 \\
\pm \\
0,01\end{array}$ & $\begin{array}{c}41,15 \\
\pm \\
1,9 \\
\end{array}$ & $\begin{array}{c}0,56 \\
\pm \\
0,01\end{array}$ & $\begin{array}{c}37,87 \\
\pm \\
2,66\end{array}$ & $\begin{array}{c}0,56 \\
\pm \\
0,01\end{array}$ \\
\hline $6-10$ & 24 & $\begin{array}{c}32,1 \\
\pm \\
1,86\end{array}$ & $\begin{array}{c}0,76 \\
\pm \\
0,01\end{array}$ & $\begin{array}{c}35,62 \\
\pm \\
1,45\end{array}$ & $\begin{array}{c}0,73 \\
\pm \\
0,01\end{array}$ & $\begin{array}{c}32,1 \\
\pm \\
1,9\end{array}$ & $\begin{array}{c}0,74 \\
\pm \\
0,01\end{array}$ & $\begin{array}{c}75,78 \\
\pm \\
2,56\end{array}$ & $\begin{array}{c}0,56 \\
\pm \\
0,01\end{array}$ & $\begin{array}{c}68,68 \\
\pm \\
2,56\end{array}$ & $\begin{array}{c}0,58 \\
\pm \\
0,01\end{array}$ & $\begin{array}{c}87,15 \\
\pm \\
2,52\end{array}$ & $\begin{array}{c}0,66 \\
\pm \\
0,01\end{array}$ & $\begin{array}{c}34,73 \\
\pm \\
2,2\end{array}$ & $\begin{array}{c}0,58 \\
\pm \\
0,01\end{array}$ & $\begin{array}{c}34,8 \\
\pm \\
2,94\end{array}$ & $\begin{array}{c}0,58 \\
\pm \\
0,01\end{array}$ \\
\hline $11-15$ & 15 & $\begin{array}{c}30,46 \\
\pm \\
1,74\end{array}$ & $\begin{array}{c}0,76 \\
\pm \\
0,01\end{array}$ & $\begin{array}{c}34,71 \\
\pm \\
1,31\end{array}$ & $\begin{array}{c}0,75 \\
\pm \\
0,01\end{array}$ & $\begin{array}{c}32,02 \\
\pm \\
2,17\end{array}$ & $\begin{array}{c}0,75 \\
\pm \\
0,01\end{array}$ & $\begin{array}{c}67,42 \\
\pm \\
1,9\end{array}$ & $\begin{array}{c}0,58 \\
\pm \\
0,01\end{array}$ & $\begin{array}{c}68,61 \\
\pm \\
1,69\end{array}$ & $\begin{array}{c}0,58 \\
\pm \\
0,01\end{array}$ & $\begin{array}{c}84,77 \\
\pm \\
1,34\end{array}$ & $\begin{array}{c}0,69 \\
\pm \\
0,01\end{array}$ & $\begin{array}{c}30,26 \\
\pm \\
1,9\end{array}$ & $\begin{array}{c}0,56 \\
\pm \\
0,01\end{array}$ & $\begin{array}{c}31,4 \\
\pm \\
2,07\end{array}$ & $\begin{array}{c}0,56 \\
\pm \\
0,01\end{array}$ \\
\hline $16-20$ & 25 & $\begin{array}{c}29,84 \\
\pm \\
1,1\end{array}$ & $\begin{array}{c}0,76 \\
\pm \\
0,01\end{array}$ & $\begin{array}{c}33,38 \\
\pm \\
1,07\end{array}$ & $\begin{array}{c}0,77 \\
\pm \\
0,01\end{array}$ & $\begin{array}{c}31,15 \\
\pm \\
1,58\end{array}$ & $\begin{array}{c}0,77 \\
\pm \\
0,01\end{array}$ & $\begin{array}{c}64,1 \\
\pm \\
1,05\end{array}$ & $\begin{array}{c}0,57 \\
\pm \\
0,01\end{array}$ & $\begin{array}{c}66,34 \\
\pm \\
1,22\end{array}$ & $\begin{array}{c}0,58 \\
\pm \\
0,01\end{array}$ & $\begin{array}{c}82,28 \\
\pm \\
2,03\end{array}$ & $\begin{array}{c}0,58 \\
\pm \\
0,01\end{array}$ & $\begin{array}{c}28,29 \\
\pm \\
1,84\end{array}$ & $\begin{array}{c}0,57 \\
\pm \\
0,01\end{array}$ & $\begin{array}{c}29,01 \\
\pm \\
1,66\end{array}$ & $\begin{array}{c}0,56 \\
\pm \\
0,01\end{array}$ \\
\hline $21-25$ & 44 & $\begin{array}{c}28,81 \\
\pm \\
0,61\end{array}$ & $\begin{array}{c}0,66 \\
\pm \\
0,01\end{array}$ & $\begin{array}{c}31,56 \\
\pm \\
1,1\end{array}$ & $\begin{array}{c}0,76 \\
\pm \\
0,01\end{array}$ & $\begin{array}{c}27,51 \\
\pm \\
1,12\end{array}$ & $\begin{array}{c}0,56 \\
\pm \\
0,01\end{array}$ & $\begin{array}{c}63,94 \\
\pm \\
0,89\end{array}$ & $\begin{array}{c}0,46 \\
\pm \\
0,01\end{array}$ & $\begin{array}{c}65,6 \\
\pm \\
0,74\end{array}$ & $\begin{array}{c}0,45 \\
\pm \\
0,01\end{array}$ & $\begin{array}{c}77,19 \\
\pm \\
0,66\end{array}$ & $\begin{array}{c}0,45 \\
\pm \\
0,01\end{array}$ & $\begin{array}{c}26,21 \\
\pm \\
0,96\end{array}$ & $\begin{array}{c}0,43 \\
\pm \\
0,01\end{array}$ & $\begin{array}{c}27,78 \\
\pm \\
0,73\end{array}$ & $\begin{array}{c}0,43 \\
\pm \\
0,01\end{array}$ \\
\hline $26-30$ & 36 & $\begin{array}{c}27,29 \\
\pm \\
0,81 \\
\end{array}$ & $\begin{array}{c}0,65 \\
\pm \\
0,01 \\
\end{array}$ & $\begin{array}{c}30,04 \\
\pm \\
0,74\end{array}$ & $\begin{array}{c}0,79 \\
\pm \\
0,01\end{array}$ & $\begin{array}{c}27,04 \\
\pm \\
0,93\end{array}$ & $\begin{array}{c}0,55 \\
\pm \\
0,01\end{array}$ & $\begin{array}{c}63,68 \\
\pm \\
0,75\end{array}$ & $\begin{array}{c}0,46 \\
\pm \\
0,01\end{array}$ & $\begin{array}{c}64,81 \\
\pm \\
0,93\end{array}$ & $\begin{array}{c}0,43 \\
\pm \\
0,01\end{array}$ & $\begin{array}{c}76,81 \\
\pm \\
0,82\end{array}$ & $\begin{array}{c}0,45 \\
\pm \\
0,01\end{array}$ & $\begin{array}{c}25,4 \\
\pm \\
0,69\end{array}$ & $\begin{array}{c}0,43 \\
\pm \\
0,01\end{array}$ & $\begin{array}{c}26,86 \\
\pm \\
0,62\end{array}$ & $\begin{array}{c}0,43 \\
\pm \\
0,01\end{array}$ \\
\hline $31-35$ & 29 & $\begin{array}{c}27,21 \\
\pm \\
0,79 \\
\end{array}$ & $\begin{array}{c}0,66 \\
\pm \\
0,01 \\
\end{array}$ & $\begin{array}{c}30,01 \\
\pm \\
0,51\end{array}$ & $\begin{array}{c}0,79 \\
\pm \\
0,01 \\
\end{array}$ & $\begin{array}{c}25,79 \\
\pm \\
0,78 \\
\end{array}$ & $\begin{array}{c}0,56 \\
\pm \\
0,01 \\
\end{array}$ & $\begin{array}{c}63,08 \\
\pm \\
1,03 \\
\end{array}$ & $\begin{array}{c}0,45 \\
\pm \\
0,01 \\
\end{array}$ & $\begin{array}{c}64,16 \\
\pm \\
1,09 \\
\end{array}$ & $\begin{array}{c}0,43 \\
\pm \\
0,01 \\
\end{array}$ & $\begin{array}{c}76,13 \\
\pm \\
0,46 \\
\end{array}$ & $\begin{array}{c}0,43 \\
\pm \\
0,01 \\
\end{array}$ & $\begin{array}{c}25,3 \\
\pm \\
0,66 \\
\end{array}$ & $\begin{array}{c}0,43 \\
\pm \\
0,01 \\
\end{array}$ & $\begin{array}{c}26,45 \\
\pm \\
0,41 \\
\end{array}$ & $\begin{array}{c}0,48 \\
\pm \\
0,01 \\
\end{array}$ \\
\hline $36-40$ & 27 & $\begin{array}{c}27,06 \\
\pm \\
0,83\end{array}$ & $\begin{array}{c}0,85 \\
\pm \\
0,01\end{array}$ & $\begin{array}{c}29,95 \\
\pm \\
0,49\end{array}$ & $\begin{array}{c}0,85 \\
\pm \\
0,01\end{array}$ & $\begin{array}{c}24 \\
\pm \\
0,85\end{array}$ & $\begin{array}{c}0,76 \\
\pm \\
0,01\end{array}$ & $\begin{array}{c}63 \\
\pm \\
0,51\end{array}$ & $\begin{array}{c}0,57 \\
\pm \\
0,01\end{array}$ & $\begin{array}{c}64,07 \\
\pm \\
0,75\end{array}$ & $\begin{array}{c}0,56 \\
\pm \\
0,01\end{array}$ & $\begin{array}{c}73,66 \\
\pm \\
1,13\end{array}$ & $\begin{array}{c}0,57 \\
\pm \\
0,01\end{array}$ & $\begin{array}{c}24,46 \\
\pm \\
0,75\end{array}$ & $\begin{array}{c}0,56 \\
\pm \\
0,01\end{array}$ & $\begin{array}{c}26,1 \\
\pm \\
0,85\end{array}$ & $\begin{array}{c}0,58 \\
\pm \\
0,01\end{array}$ \\
\hline
\end{tabular}

divisions of blood vessels of the neck and brain among the 249 examined workers of locomotive crews in the age range from 21 to 60 years. In addition, the data of Doppler examination in main group compared with normal indicators Doppler examination of intra- and extra cranial vessels in healthy employed engineers in the age range from 40 to 60 years.

The data presented in Table. 1, 2, 3. Received results indicate that the average values and their errors of indicators of Doppler examination of intra- and extra cranial vessels. The linear velocity of blood flow (average), the linear velocity of blood flow (maximum), resistance index of all examined workers of locomotive crews are reduced compared with control group, indicating that the action of harmful factors on workers leads to atherosclerotic changes and cerebrovascular pathology.

Next were have studied all the indicators of Doppler examination of neck vessels and brain in workers of locomotive crews depending on their length of service.

Received results of examination presented in Table 4 and Table 5. 
Table 6. The spread of pathological changes of cerebral in workers of locomotive crews depending on their length of service $(n=249)$

\begin{tabular}{|c|c|c|c|c|c|}
\hline \multirow[t]{2}{*}{ Length of work } & \multirow[t]{2}{*}{$\begin{array}{c}\text { Reduced elasticity } \\
\text { of blood vessels }\end{array}$} & \multicolumn{2}{|c|}{ Decreased blood flow in a.vert. } & \multirow[t]{2}{*}{$\begin{array}{l}\text { Decreased blood } \\
\text { flow in a.basil. }\end{array}$} & \multirow[t]{2}{*}{$\begin{array}{c}\text { Violation of venous } \\
\text { circulation }\end{array}$} \\
\hline & & right & left & & \\
\hline $1-5(n=49)$ & $27,77 \%$ & $23,52 \%$ & $23,52 \%$ & $23,52 \%$ & $50 \%$ \\
\hline $6-10(n=24$ & $37,5 \%$ & $37,5 \%$ & $37,5 \%$ & $37,5 \%$ & $62,5 \%$ \\
\hline $11-15(n=15)$ & $50 \%$ & $40 \%$ & $40 \%$ & $40 \%$ & $90 \%$ \\
\hline $16-20(n=25)$ & $68,42 \%$ & $60 \%$ & $60 \%$ & $60 \%$ & $94,73 \%$ \\
\hline $21-25(n=44)$ & $100 \%$ & $100 \%$ & $100 \%$ & $100 \%$ & $100 \%$ \\
\hline $26-30(n=36)$ & $100 \%$ & $100 \%$ & $100 \%$ & $100 \%$ & $100 \%$ \\
\hline $31-35(n=29)$ & $100 \%$ & $100 \%$ & $100 \%$ & $100 \%$ & $100 \%$ \\
\hline $36-40(n=27)$ & $100 \%$ & $100 \%$ & $100 \%$ & $100 \%$ & $100 \%$ \\
\hline
\end{tabular}

Table 7. The spread of pathological changes of cerebral vessels in workers of locomotive crews $(n=249)$

\begin{tabular}{|c|c|c|c|c|c|}
\hline $\mathbf{N}$ & $\begin{array}{l}\text { Reduced elasticity } \\
\text { of blood vessels }\end{array}$ & Decreas & n a.vert. & $\begin{array}{l}\text { Decreased blood } \\
\text { flow in a.basil. }\end{array}$ & $\begin{array}{c}\text { Violation of venous } \\
\text { circulation }\end{array}$ \\
\hline \multirow{2}{*}{249} & \multirow{2}{*}{$77,6 \%$} & right & left & \multirow{2}{*}{$64 \%$} & \multirow{2}{*}{$86,4 \%$} \\
\hline & & $65,6 \%$ & $65,5 \%$ & & \\
\hline
\end{tabular}

Table 8. Dynamics of thickening of the intima-media complex (TIMC) in machinists, depending on work experience $(n=249)$

\begin{tabular}{ccc}
\hline \multirow{2}{*}{ Length of work } & TIMC of a.carotis sin & TIMC of a.carotis dex \\
\cline { 2 - 3 } & & $0,74 \pm 0,2$ \\
\hline $1-5(n=49)$ & $0,75 \pm 0,2$ & $0,90 \pm 0,3$ \\
\hline $6-10(n=24$ & $0,89 \pm 0,3$ & $0,98 \pm 0,2$ \\
\hline $11-15(n=15)$ & $0,97 \pm 0,2$ & $1,20 \pm 0,3$ \\
\hline $16-20(n=25)$ & $1,19 \pm 0,3$ & $1,23 \pm 0.2$ \\
\hline $21-25(n=44)$ & $1,22 \pm 0.2$ & $1,25 \pm 0.2$ \\
\hline $26-30(n=36)$ & $1,24 \pm 0.2$ & $1,24 \pm 0,3$ \\
\hline $31-35(n=29)$ & $1,24 \pm 0,3$ & $1,28 \pm 0,3$ \\
\hline $36-40(n=27)$ & $1,26 \pm 0,3$ & \\
\hline
\end{tabular}

Based on the received results can to argue that the speed of blood flow in the neck vessels and brain in workers of locomotive crews with increasing length of service and age naturally reduced. Indicators of linear velocity of blood flow especially reduced. It is become pathological in workers, who have worked 20 years or more. These facts also confirm the presence of atherosclerotic changes in intracranial vessels in these groups of workers and the growing tendency to develop atherosclerosis with increasing length of service and age of employees. The lowest index of resistance observed in workers of locomotive crews with work experience of 21-35 years, confirming previous statements on the progressive development of vascular changes in drivers of locomotive and their assistants. Workers of locomotive crews with work experience up to 20 years have resistance index is somewhat higher, which is significantly associated with increased vascular tone of the young age. Workers of locomotive crews with work experience from 35 to 40 years have resistance index also high. In this case, change significantly associated with sclerotic changes in intra and extra cranial vessels.
We have found that spreading of pathological changes in neck and cerebral vessels among the workers of locomotive crews depend on their length of service. Results of examination showed that with increasing of length of work of locomotive drivers (according to the increasing of their age). We can see reduced elasticity of blood vessels ( $77.6 \%$ of the patients), increased violation of venous circulation (86.4\% of the patients), reduced blood flow in the basilar artery (64\%), and reduced blood flow in the right and left vertebral arteries (65.6\% and $65.5 \%$ respectively), which is directly related with length of work of workers of locomotive crews. Probably is the result of negative production factors on employees (Table 6, 7).

In addition, we have found that the thickness of the carotid intima-media complex has a direct correlation with length of service of locomotive drivers. In general, 70, $68 \%$ of drivers have overweight in the intima-media complex. The most significant thickening of the intima-media complex is observed in machinists with more than 15 years of length of work $(1,19 \pm 0,3 ; 1,20 \pm 0,3)$ in average (Table 8). 


\section{CONCLUSIONS}

1. Considering received results of examination, we found that speed of blood flow in intra- and extra cranial vessels reduced with increase the length of service of workers of locomotive crews.

2. The lowest index of resistance observed in workers of locomotive crews with work experience of 21-35 years. Workers of locomotive crews with work experience up to 20 years of resistance; index of resistance is somewhat higher, which is a result of the increase in vascular tone in young people. In workers of locomotive crews with experience of 30 to 40 years resistance index also increased, due to atherosclerotic changes of vessels.

3. During the studies found that with increasing of experience of work in workers of locomotive crews is reduced elasticity of intra- and extra cranial vessels (in $77.6 \%$ of examined). It increased violation of venous circulation (86.4\%), reduced blood flow in the basilar artery (64\% of examined), and reduced blood flow in right and left vertebral arteries $(65.6 \%$ and $65.5 \%$ respectively). It related to experience of work of the workers of locomotive crews. It is the result of impact of harmful production factors on employees.

4. In general, 70, $68 \%$ of drivers have overweight in the intima-media complex. The most significant thickening of the intima-media complex is observed in machinists with more than 15 years of length of work $(1,19 \pm 0,3$; $1,20 \pm 0,3)$ in average.

\section{REFERENCES}

1. Aaslid R., Markwalder T.M., Nornes H. Non invasive transcranial Dopplar ultrasound recording of flow velocity in basal cerebral arteries. J Neurosurg. 1982;57:769-774.

2. Sviri G.E., Ghodke B., Britz G.W. et al. Transcranial Doppler grading criteria for basilar artery vasospasm. Neurosurgery. 2006;59:360-366.

3. Kincaid M.S., Souter M.J., Treggiari M.M. et al. Accuracy of transcranial Doppler ultrasonography and single-photon emission computed tomography in the diagnosis of angiographically demonstrated cerebral vasospasm. J Neurosurg. 2009;110:67-72.

4. Arenillas J.F., Molina C.A.,Montaner J. etal. Progression and clinical recurrence of symptomatic middle cerebral artery stenosis: a long-term follow-up transcranial Doppler ultrasound study. Stroke. 2001;32:2898-2904.

5. Ducrocq X., Braun M., Debouverie M. et al. Brain death and transcranial Doppler: experience in 130 cases of brain dead patients. J Neurol Sci. 1998;160:41-46.

6. Klingelhofer J., Conrad B., Benecke R. et al. Transcranial Doppler ultrasonography of carotidobasilar collateral circulation in subclavian steal. Stroke. 1985;14:721-728.

7. Purkayastha S., Sorond F. Transcranial Doppler ultrasound: technique and application. Semin Neurol. 2012;32:411-420.

8. American College of Radiology (ACR), Society for Pediatric Radiology (SPR), Society of Radiologists in Ultrasound (SRU) AlUM practice guideline for the performance of a transcranial Doppler ultrasound examination for adults and children. J Ultrasound Med. 2012;31:1489-1500.

9. Keage H.A., Churches O.F., Kohler M. et al. Cerebrovascular function in aging and dementia: a systematic review of transcranial Doppler studies. Dement Geriatr Cogn Dis Extra. 2012;2:258-270.
10. Ringelstein E.B., Kahlscheuer B., Niggemeyer E. et al. Transcranial doppler sonography: anatomical landmarks and normal velocity values. Ultrasound Med Biol. 1990;16:745-761.

11. TegelerC.H., CrutchfieldK., Katsnelson M. etal.Transcranial Dopplervelocities in a large, healthy population. J Neuroimaging. 2013;23:466-472.

12. Alexandrov A.V., Sloan M.A., Wong L.K. et al. Practice standards for transcranial Doppler ultrasound: Part I- Test performance. J Neuroimaging. 2007;17:11-18.

13. Demirkaya S., Uluc K., BekS. et al. Normal blood flow velocities of basal cerebral arteries decrease with advancing age: a transcranial Doppler sonography study. Tohoku J Exp Med. 2008;214:145-149.

14. Tarumi T., Ayaz Khan M., Liu J. et al. Cerebral hemodynamics in normal aging: central artery stiffness, wave reflection, and pressure pulsatility. J Cereb Blood Flow Metab. 2014;34:971-978.

15. Reeves M.J., Bushnell C., Howard G. et al. Sex differences in stroke: epidemiology, clinical presentation, medical care and outcomes. Lancet Neurol. 2008;7:915-926.

16. Bentourkia M., Bol A., Ivanoiu A. et al. Comparison of regional cerebral blood flow and glucose metabolism in the normal brain: effect of aging.J Neurol Sci. 2000;181:19-28.

17. Melamed E., Lacy S., Bentin S. et al. Reduction in regional cerebral blood flow during normal aging in man. Stroke. 1980;11:31-35.

18. Tsao C.W., Seshadri S., Beiser A.S. et al. Relations of arterial stiffness and endothelial function to brain aging in the community. Neurology. 2013;81:984-991.

19. Pantano P., Baron J.C., Lebrun-Grandie P. et al. Regional cerebral blood flow and oxygen consumption in human aging. Stroke. 1984;15:635-641.

20. Levin B.E., Llabre M.M., Dong C. et al. Modeling metabolic syndrome and its association with cognition: the Northern Manhattan study. J Int Neuropsychol Soc. 2014;20:951-960.

21. Gordon Perue G.L., Narayan R., Zangiabadi A.H. et al. Prevalence of vertebral artery origin stenosis in a multirace-ethnic posterior circulation stroke cohort: Miami Stroke Registry (MIAMISR) Int J Stroke. 2015;10:185-187.

22. Sacco R.L., Kargman D.E., Gu Q. et al. Race-ethnicity and determinants of intracranial atherosclerotic cerebral infarction: the Northern Manhattan Stroke Study. Stroke. 1995;26:14-20.

23. Schmidt E.A., Piechnik S.K., Smielewski P. et al. Symmetry of cerebral hemodynamic indices derived from bilateral transcranial Doppler. J Neuroimaging. 2003;13:248-254.

24. Diehl R.R., Diehl B., Sitzer M. et al. Spontaneous oscillations in cerebral blood flow velocity in normal humans and in patients with carotid artery disease. Neurosci Lett. 1991;127: 5-8.

25. Huber P. Krayenbühl and Yasargil's cerebral angiography. Thieme, Stuttgart. 1982: 10-15.

26. Newell D.W., Aaslid R., Lam A. et al. Comparison of flow and velocity during dynamic autoregulation testing in humans. Stroke 1994; 25: 793-797.

27. Patel D.J., Vaishnav R.N. Basic hemodynamics and its role in disease processes. University Park Press, Baltimore. 1980:17-37.

28. Ring B.A., Waddington M.M. Intraluminal diameters of the intracranial arteries. Vasc Surg 1. 1967: 137-151.

29. Rossitti S., Löfgren J. Vascular dimensions of the cerebral arteries follow the principle of minimum work. Stroke 1993; 24: 371-377.

30. Silvestrini M., Cupini L.M., Matteis M. et al. Bilateral simultaneous assessment of cerebral flow velocity during mental activity. J Cereb Blood Flow Metab. 1994.14: 643-648.

31. Sitzer M., Knorr U., Seitz J. Cerebral hemodynamics during sensorimotor activation. J Appl Physiol. 1994; 77: 2804-2811. 
32. Smiesko V., Johnson P.C. The arterial lumen is controlled by flow-related shear stress. News Physiol Sci. 1993;8: 34-38.

33. Sorterberg W. Methodological and technical advances in transcranial Doppler ultrasonography. PhD Thesis, 0slo. 1993:123-143,

34. Stehbens W.E. Pathology of the cerebral blood vessels. Mosby, Saint Louis, 1972; 471-558.

The work is a fragment of scientific research - "Impact of negative production factors on the health of workers of locomotive crews, directions of improvement of medical examinations" (code № 0110U006816), conducted by the employees of Bogomolet's National Medical University. This work did with the involvement of the employees of the 2Kyiv Railway Clinical Hospital №2 of Branch "Health Center» of the Joint Stock Company "Ukrainian Railway» and the branch program "Health of railway workers on the South-Western Railway (prevention and treatment of cardiovascular and cardiovascular diseases)».

\section{ORCID and contributionship:}

Oleksandr Yu. Aleksiichuk : 0000-0002-6039-2574 ${ }^{\mathrm{A}}$

Nataliia Yu. Tkachyshyna: 0000-0001-9828-4977 ${ }^{B}$

Irina V. Dumka: 0000-0002-6482-985X ${ }^{\mathrm{C}}$

Olha M. Arustamian: 0000-0001-7733-9887 D

Volodymyr S. Tkachyshyn: 0000-0003-2217-7416 ${ }^{E}$

Vitalii E. Kondratiuk: 0000-0002-4891-2338 ${ }^{F}$

\section{Conflict of interest:}

The Authors declare no conflict of interest.

\section{CORRESPONDING AUTHOR}

Olha M. Arustamian

Bogomolet's National Medical University

13 T. Shevchenko Boulevard, 01601 Kyiv, Ukraine

tel: +380509169326

e-mail: olga-zozulya@ukr.net

Received: 22.01 .2020

Accepted: 31.08 .2020

A - Work concept and design, B - Data collection and analysis, C - Responsibility for statistical analysis, D-Writing the article, $\mathbf{E}$-Critical review, $\mathbf{F}$ - Final approval of the article 